\title{
Quantization and Time
}

\author{
A. A. Abrikosov, Jr. ${ }^{\mathrm{a}}$ and Ennio Gozzi ${ }^{\mathrm{b}}$ \\ aTEP, Bol. Cheremushkinskaya, 25; Moscow, 117259 Russia. \\ ${ }^{b}$ Department of Theoretical Physics, University of Trieste \\ Strada Costiera 11, Miramare-Grignano 34014, Trieste \\ and INFN, Trieste, Italy.
}

Starting from a functional formulation of classical mechanics, we show how to perform its quantization by freezing to zero two Grassmannian partners of time.

\section{INTRODUCTION}

This conference is centered on quantum gravity which seems to be one of the most difficult and unsolved problems that physicists have faced in this century. The two components of the problem are quantum mechanics and gravity. Physicists have worked mostly on the second one that is gravity. They have tried to modify gravity by going to supergravity, strings and M-theory etc. with the hope of obtaining a theory that, after quantization, would be free of the ultraviolet problems of standard gravity. We wonder why they have not thought more about the first component of the problem, namely about quantum mechanics (QM). By "thought more" we do not mean problems like the measurement issue etc., but we mean the geometrical aspects of quantum mechanics. After all, before marrying quantum mechanics to gravity which is the queen of geometrical theories, one should better understand quantum mechanics from a more geometrical point of view. We mean the following. Quantum mechanics is usually formulated via Hilbert space kind of tools and space-time apppears like a secondary concept. This, we think, creates hidden conceptual difficulties in applying quantum mechanics to space-time theories such as gravity. The standard procedure itself of quantization seems to have nothing to do with space-time. In this paper we shall not change this picture. In particular we shall not suggest things like quantization of time as one could have wrongly guessed from the title of the talk. What we will show is that, by formulating classical mechanics (CM) in a more modern way,[5], the standard quantization rules become equivalent to freezing to zero some Grassmannian partners of time. This is how "space-time" concepts enter the picture of quantization in our approach.

At the beginning of a new century it seems quite timely to start rethinking about quantum mechanics. Remember how the last century started: on Dec.14th, 1900 M. Planck presented the paper, [1], which contained the idea of the Planck constant and that day was called by A. Sommerfeld the "birthday of quantum mechanics", [2].

\section{FUNCTIONAL APPROACH TO CLASSICAL MECHANICS}

The "modern" formulation of classical mechanics $(\mathrm{CM})$ mentioned in the introduction is actually a functional approach to the old operatorial version of CM proposed by Koopmann and von Neumann, [3]. These authors, instead of using the Hamiltonian and the Poisson brackets for the classical evolution of a system, used the wellknown Liouville operator, $\hat{L} \equiv \frac{\partial H}{\partial p} \frac{\partial}{\partial q}-\frac{\partial H}{\partial q} \frac{\partial}{\partial p}$, and the associated commutators. One can even generalize their formalism to higher forms and get the Lie derivative of the Hamiltonian flow, [4]. It was shown in [5] that the operatorial formalism mentioned above could have a functional or path integral counterpart even at the level of pure clas- 
sical mechanics. The path integral [5] basically assigns weight one to classical paths and zero to others.

Let us denote by $\mathcal{M}$ our phase space with $2 n$ phase-space coordinates $\varphi^{a}=\left(q^{j}, p^{j}\right)$ (the index " $a$ " spans both $q$ 's and $p$ 's) and by $H(\varphi)$ the Hamiltonian of the system. Then the classical probability $P\left(\varphi_{f}, t_{f} \mid \varphi_{i}, t_{i}\right)$ of going from the initial phase-space configuration $\varphi_{i}$ at time $t_{i}$ to the final one $\varphi_{f}$ at time $t_{f}$, is nothing but:

$P\left(\varphi_{f}, t_{f} \mid \varphi_{i}, t_{i}\right)=\delta\left[\varphi_{f}-\tilde{\phi}_{c l}\left(t_{f} ; \varphi_{i}, t_{i}\right)\right]$,

where $\tilde{\phi}_{c l}\left(t ; \varphi_{i}, t_{i}\right)$ is the classical trajectory starting at the moment $t_{i}$ from the phase-space point $\varphi_{i}$. These trajectories are solutions of the Hamilton equations of motion: $\varphi^{a}=\omega^{a b} \frac{\partial H}{\partial \phi^{b}}$ with $\omega^{a b}$ being the standard symplectic matrix. Slicing the time interval $t_{f}-t_{i}$ into $\mathrm{n}$ smaller ones and doing some manipulations on the Dirac delta in the RHS of eq.(1), one can rewrite the probability as a path integral (for more detail see ref.[5]):

$P\left(\varphi_{f}, t_{f} \mid \varphi_{i}, t_{i}\right)=\int \mathcal{D} \mu \exp i \int_{t_{i}}^{t_{f}} \widetilde{\mathcal{L}} d t$,

where $\mathcal{D} \mu \equiv \mathcal{D}^{\prime \prime} \varphi^{a} \mathcal{D} \lambda_{a} \mathcal{D} c^{a} \mathcal{D} \bar{c}_{a}$. The notation $\mathcal{D}^{\prime \prime}$ indicates that first and last integrations over $\varphi$ are not done. The geometrical meaning of the $6 n$ auxiliary variables $\lambda_{a}, c^{a}, \bar{c}_{a}$ (where the $c$ and $\bar{c}$ variables are Grassmannian) is explained in detail in [5]. The Lagrangian $\widetilde{\mathcal{L}}$ in eq.(2) and the associated Hamiltonian are:

$\widetilde{\mathcal{L}}=\lambda_{a}\left[\dot{\varphi}^{a}-\omega^{a b} \partial_{b} H\right]+i \bar{c}_{a}\left[\delta_{b}^{a} \partial_{t}-\omega^{a c} \partial_{c} \partial_{b} H\right] c^{b}$

$\widetilde{\mathcal{H}}=\lambda_{a} \omega^{a b} \partial_{b} H+i \bar{c}_{a} \omega^{a c}\left(\partial_{c} \partial_{b} H\right) c^{b}$

The connection with the operatorial formalism, [3], can be established immediately and has been thoroughly studied in ref.[5]. There it was shown that the variables $\varphi^{a}$ and $c^{a}$ can be realized as multiplicative operators while $\lambda_{a}$ and $\bar{c}_{a}$ are derivative ones. So one could define a basis $|\varphi, c\rangle$ as $\hat{\varphi}|\phi, c>=\varphi| \varphi, c>$ and $\hat{c}|\varphi, c>=c| \varphi, c>$ and the kernel of propagation between these states would have the following path integral representation:

$$
\begin{aligned}
& <\varphi_{f}, c_{f} ; t_{f} \mid \varphi_{i}, c_{i} ; t_{i}>= \\
& =\int_{\left(\varphi_{i}, c_{i}\right)}^{\left(\varphi_{f}, c_{f}\right)} \mathcal{D} \mu^{\prime} \exp i \int \widetilde{\mathcal{L}} d t
\end{aligned}
$$

where $\mathcal{D} \mu^{\prime}$ is the same measure as in eq.(2) but without initial and final integrations over $c^{a}$. The relation between the probability, eq.(2), and the amplitude, eq.(4), is:

$$
\begin{aligned}
& P\left(\varphi_{f}, t_{f} \mid \varphi_{i}, t_{i}\right)= \\
& \quad K \int\left|<\varphi_{f}, c_{f} ; t_{f}\right| \varphi_{i}, c_{i} ; t_{i}>\left.\right|^{2} d c_{i} d c_{f} ;
\end{aligned}
$$

where $K$ is an appropriate constant. The reader may be puzzled why both the probability, eq.(2), and the amplitude, eq.(4), have the same path integral representation. This happens because both of them evolve via the Liouville operator that contains only first order derivatives (differently than the second order Schrödinger operator).

Now we would like to address the question of how we should quantize CM once it is formulated this way? Some attempts to answer it have been done in [6]. In the next section we shall put forward a new idea.

\section{QUANTIZATION AND GRASSMAN- NIAN PARTNERS OF TIME.}

The whole $8 n$ variables $\left(\phi^{a}, c^{a}, \lambda_{a}, \bar{c}_{a}\right)$ can be assembled into a single variable as follows. First we introduce two Grassmannian partners, $\theta$ and $\bar{\theta}$, of the standard time $t$. This converts the base space (which is $t$ ) to a superspace: $(t, \theta, \bar{\theta})$. We can then put together all variables $\left(\phi^{a}, c^{a}, \lambda_{a}, \bar{c}_{a}\right)$ in a single "super"-field variable $\Phi^{a}$ defined as follows:

$$
\begin{aligned}
\Phi^{a}(t, \theta, \bar{\theta}) \equiv \phi^{a}(t) & +\theta c^{a}(t)+ \\
& +\bar{\theta} \omega^{a b} \bar{c}_{b}(t)+i \bar{\theta} \theta \omega^{a b} \lambda_{b}(t) .
\end{aligned}
$$

The superfield $\Phi^{a}$ is a very useful tool and, unexpectedly, it brings to light some interplay between $\mathrm{CM}$ and QM. Let us for example replace the variable $\varphi^{a}$ with the superfield $\Phi^{a}$ into the original Hamiltonian $H\left(\varphi^{a}\right)$ of our classical system. Expanding it in $\theta$ and $\bar{\theta}$ we get:

$$
\begin{aligned}
H\left(\Phi^{a}\right)=H\left(\varphi^{a}\right) & +\theta \frac{\partial H}{\partial \varphi^{a}} c^{a}+ \\
& +\bar{\theta} \frac{\partial H}{\partial \varphi^{a}} \omega^{a b} \bar{c}_{b}+i \theta \bar{\theta} \widetilde{\mathcal{H}}
\end{aligned}
$$


and doing the same with the old action associated with $H(\varphi)$, that is $S=\int(p \dot{q}-H) d t$, we get:

$S\left[\Phi^{a}\right]=S\left[\varphi^{a}\right]+\theta \mathcal{T}+\bar{\theta} \mathcal{V}+i \theta \bar{\theta}[\widetilde{\mathcal{S}}+($ s.t. $)]$.

Here $\mathcal{V}$ and $\mathcal{T}$ are functionals that are of no interest for the moment and the (s.t.) is a surface term: (s.t.) $=\left.\frac{1}{2}\left(\lambda_{a} \varphi^{a}+i \bar{c}_{a} c^{a}\right)\right|_{t_{i}} ^{t_{f}}$. The last term, $\widetilde{\mathcal{S}}=\int \widetilde{\mathcal{L}} d t$, is the action which enters our $\mathrm{CM}$ path integral, eq.(2), while the first term, $S(\varphi)$, is the action of the QM path integral. The occurrence, in the same supermultiplet, of both the QM and CM actions cannot be an accident and must have some intriguing geometrical meaning.

Let us first notice that the classical weight $\widetilde{\mathcal{S}}$ can be obtained from the action $S[\Phi]$ :

$\widetilde{\mathcal{S}}=\int S[\Phi] i d \theta d \bar{\theta}-($ s.t. $)$.

This implies that eq.(4) can be rewritten as:

$$
\begin{aligned}
& <\varphi_{f}, c_{f} ; t_{f} \mid \varphi_{i}, c_{i} ; t_{i}>= \\
& \quad \int \mathcal{D} \Phi \exp i \int S[\Phi] i d \theta d \bar{\theta}-(\text { s.t. }) ;
\end{aligned}
$$

where we have formally written the measure $\mathcal{D} \mu^{\prime}$ of eq.(4) in terms of superfields $\Phi$. We can get rid of the surface term (s.t.) in the RHS of eq.(10) by performing a proper Fourier transform of both sides. This Fourier transform involves some of the variables $\varphi^{a}$ and their "momenta" $\lambda_{a}\left(\lambda_{a}\right.$ are momenta if one uses the $\widetilde{\mathcal{L}}$ as lagrangian.) The result is that the $(q, p)$-variables are replaced by the $\left(q, \lambda_{p}\right)$ and $\left(c^{q}, \bar{c}_{p}\right)$ take the place of $\left(c^{q}, c^{p}\right)$. The index " $q$ " stands for the first $n$ indices " $a$ " in $\left(\phi^{a}, c^{a}, \lambda_{a}, \bar{c}_{a}\right)$, and " $p$ " stands for the second ones. After the Fourier transform we obtain:

$$
<\Phi_{f}^{q} ; t_{f} \mid \Phi_{i}^{q} ; t_{i}>=\int \mathcal{D} \Phi e^{i \int S[\Phi] i d \theta d \bar{\theta}},
$$

where the LHS of (11) stands for

$$
<q_{f}, \lambda_{p, f}, c_{f}^{q}, \bar{c}_{p, f} ; t_{f} \mid q_{i}, \lambda_{p, i}, c_{i}^{q}, \bar{c}_{p, i} ; t_{i}>
$$

which is the Fourier transform of the LHS of eq. (10). Note that the variables entering the bra and ket of (12) are exactly those making up the superfield $\Phi^{q}$ in the LHS of (11). At this point we cannot avoid noticing the formal analogy between the classical relation (11) and the standard quantum one which is:

$$
<\varphi_{f}^{q} ; t_{f} \mid \varphi_{i}^{q} ; t_{i}>=\int \mathcal{D} \varphi e^{\frac{i}{\hbar} S[\varphi]} .
$$

One goes from eq. (11) to (13) by letting $\theta, \bar{\theta}$ go to zero: $\lim _{\theta, \bar{\theta} \rightarrow 0} \Phi^{q}=\varphi^{q}=q$. The whole quantization procedure can then be formally represented as:

$$
\begin{aligned}
& <\Phi_{f}^{q}\left|\int \mathcal{D} \Phi \quad \exp \quad i \int S[\Phi] i d \theta d \bar{\theta}\right| \Phi_{i}^{q}> \\
& \text { quantization : } \Downarrow \quad \theta, \bar{\theta} \rightarrow 0 \\
& <q_{f}\left|\int \mathcal{D} \varphi \quad \exp \frac{i}{\hbar} S[\varphi]\right| q_{i}>.
\end{aligned}
$$

One may wonder how $\hbar$ appears. First let us notice from eqs.(8) and (9) that the dimension of the volume element $d \theta d \bar{\theta}$ is the inverse of action. A correct procedure of sending $\theta, \bar{\theta}$ to zero must respect the dimension. This may be done by sticking $-\frac{i}{\hbar} \delta(\theta) \delta(\bar{\theta})$ into the $d \theta d \bar{\theta}$ integration in the exponent in the RHS of (11). The procedure can be made more rigorous and precise, [7], by introducing the generating functional $Z_{c l}[J]$ associated to the classical path-integral, (11), where $J$ is a supercurrent coupled to the superfield $\Phi$. By performing a proper limit of $\theta, \bar{\theta} \rightarrow 0$, it is possible to show that $Z_{c l}[J]$ goes into the quantum generating functional $Z[j]$ where $j$ is the component of the supercurrent $J$ coupled to the variable $\varphi$.

The procedure that we have outlined above belongs to the same category as geometric quantization, [8]. The reason to say this is that geometric quantization brings us from the Lie derivative of the Hamiltonian flow to the Schrödinger operator. We achieved the same by our procedure: we basically managed to go from the weight $\widetilde{\mathcal{S}}$ of the classical path integral (and the associated $\widetilde{\mathcal{H}}$ which is the classical Lie derivative, [5]) to the weight $S(\varphi)$ that generates the Schrödinger operator. All the complex machinery of geometric quantization, [8], is basically encapsulated in the $\theta, \bar{\theta} \rightarrow 0$ procedure. The reader expert in geometric quantization will notice that the issue of "polarization" was naturally incorporated via the surface terms, (s.t.), of eq.(10) which magically 
appeared thanks to the superfield formalism. The surface terms plus the $\theta, \bar{\theta} \rightarrow 0$ procedure naturally bring us from $(q, p)$-von Neumann states to $q$-Schrödinger states and simultaneously generate the correct path integral weight. The beauty of the procedure is that it generates the correct states and the correct weight at once. Other polarizations can be obtained in a similar way, [7].

\section{CONCLUSIONS}

If quantization is equivalent to sending the Grassmannian partners of time to zero, we should ask ourselves what this means from a physical point of view. First we should understand the physical meaning of the variables $\theta, \bar{\theta}$. In our formalism, [5], all Grassmannian variables in the target-space are forms, for example $c^{a} \sim d \varphi^{a}$. Almost the same happens at the base-space level,[7], where $\theta \sim \sqrt{d t}$. Thus the projector, $\frac{\theta \bar{\theta}}{\hbar}$, that we are putting in to pass from CM to QM is equivalent to $(\Delta t) \sim \hbar$. This is a well-known fact [9] which is basically the central feature QM.

The second point that we are exploring, [7], is the following. In our formulation of CM, [5], we have found several universal symmetries relating the bosonic variables to the Grassmannian ones in the target space. The same symmetries have an image also at the level of the base-space $(t, \theta, \bar{\theta})$. In $\mathrm{QM}$, where $\theta, \bar{\theta}=0$, all these symmetries are lost. Now let us ask ourselves if there is a universal symmetry of CM that is always lost in QM. The answer is yes !, [10], and it corresponds to the universal symmetry of rescaling the over-all action. That symmetry can be put in the form of a transformations relating the $\theta$ with the t, [7].

All this indicates that some geometrical structure, even if Grassmannian, is behind the quantization problem.

\section{Acknowledgments}

A. A. is glad to express his gratitude to the Department of Theoretical Physics of the University of Trieste where a part of the work was done. E. G. would like to thank V. De Alfaro, for inviting him to deliver this talk, M. Berry, E. Deotto, D. Mauro, M. Reuter for helpful discussions and
F. Legovini for much needed help with $\mathrm{LAT}_{\mathrm{E}} \mathrm{X}$. This work was supported by grants from MURST, NATO and INFN.

\section{REFERENCES}

1. M. Planck, Vehr.D.Deutsch. Phys.Ges. (2) 2, (1900) 237-245;

2. A. Sommerfeld, Atombau und Spektrallinien, Braunschweig:Fr. Vieweg and Sohn (1919);

3. B.O.Koopman, Proc.Nat.Acad.Sc i. USA 17 (1931)315; J.von Neumann Ann.Math. 33 (1932) 587 ;

4. R.Abraham and J.Marsden, Foundations of Mechanics, Benjamin, New York, 1978;

5. E.Gozzi, M.Reuter, W.D.Thacker, Phys. Rev.D 40 (1989) 3363; Phys.Rev.D 46, 2 (1992) 757; A.A.Abrikosov,(Jr.) Nucl. Phys. B382 (1992) 581; E.Gozzi, M.Reuter, Phys. Lett. B 233 (3.4) (1989) 383; Phys. Lett. B 240 (1,2) (1990) 137; E.Gozzi, M.Regini, Hepth-9903136, Phys.Rev. D in press; E.Gozzi, D.Mauro, Hep-th-9907065, Jour. Math. Phys. in press.

6. E.Gozzi, Phys. Lett.A $202(5,6)(1995)$ 330;

E.Gozzi, Nucl. Phys.(Proc.Supp) 57 (1997) 223; M.Reuter, Int. Jour. Theor. Phys.A 13 (1998) 3835.

7. E.Gozzi et al. to appear;

8. N.Woodhouse, Geometric quantization, Claredon Press, Oxford, 1980.

9. B.Sakita, Quantum Theory of Many-Variable Systems and Fields, World Scientific, Singapore, 1985.

10. E.Gozzi, Phys.Lett.B 158 489;Ibid.Err.Phys.Lett.386 (1996) 495.
$(1985)$ 